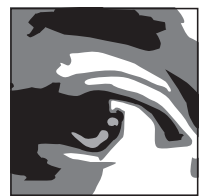

\title{
Mending the Cracks: A Case Study in Using Technology to Assist with Transitional Care for Persons with Dementia
}

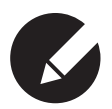

Kim Ritchie, RN, MN, PhD(c), GNC(c)

Professional Practice Leader, Professional Practice Ontario Shores Centre for Mental Health Sciences Whitby, ON

Andra Duff-Woskosky, BSc., BHSc.(PT), MHSc.(Admin.), CHE Administrative Director, Geriatric \& Neuropsychiatric Program Ontario Shores Centre for Mental Health Sciences Whitby, ON

Sarah Kipping, RN, BSc., MSN, CPMHN(C) Professional Practice Leader, Professional Practice Ontario Shores Centre for Mental Health Sciences Whitby, ON

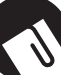

\section{Abstract}

Transitions between hospital and community are particularly challenging for vulnerable adults experiencing behavioural and psychological symptoms (BPSD) of dementia. Too often, miscommunication results in triggering a recurrence of disruptive behaviours leading to frustration of staff and families. As part of the implementation of Health Quality Ontario (HOO) Quality Standards, this project

This article originally appeared in Nursing Leadership 30(3) September 2017: 54-62.doi:10.12927/ cjnl.2018.25385. <https://www.longwoods.com/content/25385/nursing-leadership/mending-thecracks-a-case-study-in-using-technology-to-assist-with-transitional-care-for-persons-wi $>$. 
involved improving transitions using an electronic-based care plan on a 23-bed geriatric dementia unit in a mental health hospital. "My Dementia Careplan," is an interprofessional care plan that was developed in the electronic medical record (EMR) to enhance communication of information between healthcare providers when patients are being discharged to the community. It is written from the patient's perspective in collaboration with the family and interprofessional team. It describes strategies to manage behavioural challenges along with the standardized tools to objectively monitor progress. This care planning will help to support transition of knowledge between hospital and community.

\section{Background}

Improving transitional care in adults with

dementia has been identified as a priority for improving health system outcomes

(Chenoweth et al. 2015). Transitional care is defined as, "the actions involved in coordinating care for patients as they move through the health care system" (Epstein-Lubow et al. 2010). Research has found that care transitions in adults with dementia are associated with an increase in poor outcomes such as early readmission, mortality, change in behaviour or functional status and caregiver satisfaction (Tsilimingras et al. 2009). Despite the need to identify ways to improve transitional care for adults with dementia, there have been few transitional care practices developed for this population (Fortinsky and Downs 2014).

As dementia progresses, impaired cognition reduces an individual's ability to communicate their needs or comprehend what is going on around them, making these patients very vulnerable during transitions (Kable et al. 2015). Risk increases when there are multiple transitions from hospital to community because of the need for symptom management for behavioural and psychological symptoms of dementia (BPSD) such as verbal agitation, intrusiveness, wandering, resisting care or physical aggression (Ray et al. 2015). For these reasons, it has been found that the complex nature of dementia requires transitional care with both higher levels of coordination and improved communication during the discharge process to improve outcomes (Chenoweth et al. 2015; EpsteinLubow et al. 2010).

Electronic medical records (EMR) have been found to enhance communication during transitions in care (Tsilimingras and Bates 2008). The use of an EMR-based interprofessional plan of care has been suggested as a tool that can improve communication during transitions (Dykes et al. 2014). Traditionally, the plan of care has been developed by a single discipline within a specific healthcare setting and as patients are transferred from one setting to another, a new plan of care is developed (Dykes et al. 2014). During these transitions, gaps in communication including incomplete, inaccurate or delayed information are associated with threats to patient safety (Dykes et al. 2014; Tsilimingras et al. 2009). The use of an EMR-based interprofessional plan of care has several advantages because it is developed by multiple disciplines and can pull together various elements that are relevant to the new care setting (Dykes et al. 2014). This provides information to the new setting that is accurate and readily available.

Health Quality Ontario (HQO) has developed Quality Standards for the Behavioural Symptoms of Dementia care for patients in hospitals and residents in longterm care homes (Health Quality Ontario 2016). This quality standard identifies $14 \mathrm{key}$ 
quality statements as having a significant potential for improvement in the quality of care for older adults living with dementia and experiencing symptoms of agitation or aggression. In 2016, Ontario Shores Centre for Mental Health Sciences committed a corporate goal to implement the HQO Quality Standards for the Behavioural Symptoms of Dementia Care. As part of this larger project, there was a focus on creating dementia-specific strategies to enhance seamless communication in an effort to improve transitional care of older adults with dementia as they are discharged back to the community. To achieve this goal, two HQO quality standards were used to provide the benchmark to improve transitional care; Individualized Care Plan and Transitions in Care. The objectives to meet these standards were to: (1) customize the EMR to create a dementiaspecific interprofessional care plan; and (2) use the EMR to facilitate an accurate and timely transition of information to community healthcare providers. This case study describes the development of these objectives in a specialized mental health hospital to meet the HQO Quality Standards and improve transitional care in adults with dementia experiencing BPSD. Key success factors and challenges to implementation will be presented.

\section{Intervention}

Ontario Shores Centre for Mental Health Sciences (Ontario Shores) is a public teaching hospital that specializes in a range of specialized assessment and treatment services to individuals experiencing complex mental illness. In 2009, Ontario Shores implemented an electronic medical record (EMR) and has since become the first Canadian hospital to achieve HIMSS (Healthcare Information and Management System Society) Stage 7 status. HIMSS developed an eightstage Electronic Medical Record Adoption Model (EMRAM) to reflect the EMR capabilities in hospitals ranging from completely paper-based (score 0 ) to highly advanced digital patient record (score 7) (Van Poelgeest et al. 2015). The Geriatric Dementia Unit (GDU) at Ontario Shores is a 23-bed inpatient unit that specializes in the mental health needs of individuals living with dementia and experiencing challenging behaviours such as agitation and aggression. Ontario Shores is guided by the overarching philosophy of the Interprofessional Collaborative Recovery Model based on the principles of empowerment, hope, recovery, collaboration, identity, responsibility and meaning in life (Oades et al. 2009).

A documentation working group was established to focus on meeting the two HQO standards. It reported to an overall advisory group that was responsible for implementing all 14 of the HQO standards. The documentation working group comprised an interprofessional team of eight individuals, including: professional practice leaders (2), clinical nurse specialist in dementia (1), nursing (RN/RPN) (2), decision support (1), social work (1) and a clinical manager (1). The other working groups involved in the overall implementation of the HQO standards focused on: physician processes, education and training, outpatient and behavioural approach.

\section{Objective 1: Dementia-specific interprofessional care plan}

One of the key goals of the team was to design one dementia-specific care plan that could be documented against by the entire interprofessional team, including physicians, nurses (RN and RPN), occupational therapy and others. This was a change from the previous care plan functionality that because of the EMR design limited documentation accessibility to nursing and allied health professionals. The previous care plan also had multiple care plans for different types of medical and psychiatric goals. The aim of having an interprofessional care plan 
was to promote effective communication among the team and enhance consistency in interventions. In addition, by integrating all medical and psychiatric care into one care plan, it would be simpler for staff to use and navigate.

To inform the content of a dementiaspecific care plan, the working group reviewed and appraised the recommendations from The National Institute for Health and Care Excellence (NICE) guideline for, "Dementia ... supporting people with dementia and their carers in health and social care" (National Collaborating Centre for Mental Health [UK] 2007). Specific recommendations from this guideline were selected by the working group relevant to the needs of patients in a tertiary care specialized dementia unit (i.e., recommendations related to prevention or early identification were not reviewed). A gap analysis compared current care plan content and processes to the NICE guideline recommendations. Current care plan content and processes that were not aligned with the NICE recommendations were identified and a plan was developed to address these gaps and to implement them into the EMR.

A second area of focus was to integrate standardized clinical practice guideline (CPG) assessment tools into the care plan. Although the use of standardized assessment tools was well established on the unit, they were still stand-alone templates in the EMR or paper form. By customizing the EMR to integrate standardized assessment tools scores into the care plan, the results of the standardized assessment tools could directly inform the care plan. Based on the CPG assessment, standardized tools were identified, and permissions were obtained to reproduce them in the EMR. Requirements for the frequency of tool completion were built into the EMR based on the individual tool specifications and the working group consensus where appropriate (e.g., Cornell scale is assigned a frequency for completion of every 7 days). This design provided a prompt whereby clinical staff would be notified when a standardized tool required completion.

A third focus of the care plan development was to develop a structured process to include family and Substitute Decision Makers (SDMs) in the phases of plan development, plan review and information to be transferred at discharge. Prior to this project, families and SDMs were key informants in the development of care plan goals; however, the working group determined that this process needed to be formalized with the use of the EMR to ensure consistency. As a result, an EMR template was developed to guide family meetings and to document their input and consent into the care plan.

\section{Objective 2: Ensure the accurate and timely transition of knowledge to community healthcare providers}

To provide the most accurate information, a new prompt needed to be added to the EMR to notify the interprofessional team to update the care plan just prior to discharge. Coordination of the discharge required a template to be built into the EMR with accountability for completion assigned to the social worker who was already the most involved in the discharge process. This template was designed primarily for documentation and communication of key care plan details regarding the transition between the team, community long-term care (LTC) and family/SDM in the form of an in-person or telephone conference. It also prompted the transfer of the care plan immediately upon discharge to enrolled community LTCs and the family/SDM through the Patient Portal.

\section{Results}

Objective 1: Dementia-specific interprofessional care plan

"My Care Plan: Dementia" was the name 
Table 1. My care plan: dementia assessment and care planning domains

\begin{tabular}{|l|}
\hline My behavioural needs \\
\hline My physical health issues \\
\hline My risk needs \\
\hline My activities of daily living (ADL) needs \\
\hline My quality of life/risk \\
\hline My caregiver needs \\
\hline My transition to the community status \\
\hline Care plan reviewed \\
\hline Care plan updated \\
\hline
\end{tabular}

Table 2. My care plan: dementia standardized assessment tools

\begin{tabular}{|l|l|}
\hline $\begin{array}{l}\text { Standardized assessment tools incorporated into my } \\
\text { care plan: dementia }\end{array}$ & Description \\
\hline Barthel Index & Activities of daily living \\
\hline PAINAD & Pain \\
\hline Choking & Choking risk \\
\hline Falls & Falls risk \\
\hline Braden scale & Predicting pressure ulcer risk \\
\hline Holden & Communication scale \\
\hline CAM & Delirium screening \\
\hline Cornell & Depression in dementia \\
\hline NPI-NH & Neuropsychiatric symptoms \\
\hline Zarit & Caregiver burden \\
\hline
\end{tabular}

given to the new EMR interprofessional care plan that can be accessed and documented by the entire interprofessional team (nurses, allied health professionals and physicians). It is built as a single document that incorporates assessment and care planning within seven domains (Table 1). To align with the Interprofessional Recovery Model, the care plan was written in the first person to empower individuals with dementia to actively identify and participate into their care plan. The care plan is reviewed by nursing staff each shift and by the interprofessional team every 28 days during pre-scheduled patient conferences.

The gap analysis relative to the NICE guidelines, identified several areas that required action to be in aligned with same.
The additional items that were relevant to the development of the care plan included:

- expansion of admission documentation;

- creation of an incontinence template;

- addition of pain assessment;

- cognitive testing;

- build of interprofessional consult referral;

- carer assessment.

Several other items in the NICE guidelines such as capacity assessment, medical investigation and mental status exam had previously been integrated into the EMR and did not require any further action. 
Standardized tools were reviewed and chosen by the working group based on evidence of reliability and validity, feasibility, ability to reproduce in the EMR and cost. After receiving permissions, several standardized tools were reproduced in the EMR and integrated into the assessment (Table 2).

A family meeting template was developed to capture relevant information from family meetings and to collaborate in the care plan and treatment goals. In addition, the family meeting template provides an area to document that the care plan has been reviewed with family/SDM. Family meetings are conducted within the first two weeks of admission, as needed for the duration of hospitalization and then again prior to discharge.

Objective 2: Ensure the accurate transition of information to community healthcare providers

A new pre-discharge order set was developed in the EMR that includes a repeated list of standardized assessment measurement to inform readiness for discharge. The scores from the repeated standardized assessment tools are automatically updated in My Care

Plan: Dementia and sent to the LTC facility by printed form, by fax or by patient portal.

This process provides LTC providers with accurate and updated patient information. The transition process is initiated early in admission with a meeting (telephone or in-person) scheduled with the discharge facility to begin planning for discharge.

Another meeting is arranged 1-2 weeks prior to discharge with the patient, family, LTC and community supports (Community Care Access Centre [CCAC], Ontario Shores Community Outreach Team, Psychogeriatric Resource Consultants). This final meeting provides an opportunity to transfer knowledge, progress, interventions and care strategies, and to identify proactive approaches to ensure a smooth transition.
To facilitate discharge, a new discharge checklist template was created. The discharge checklist is completed by the social worker with prompts to have a predischarge conference if required (including LTC and family/ SDM) and to share information with family and the LTC providers. Completion of the checklist triggers the Health Information Management (HIM) department to send a current copy of My Care Plan: Dementia to the LTC facility and prompts the sending of physician discharge summaries.

\section{Discussion}

This case study describes the development of a dementia-specific care plan and transition process using the EMR to improve communication during transitions from hospital to community. Improving communication between healthcare facilities has been identified as an important mechanism to reduce gaps when transitioning individuals with dementia (Chenoweth et al. 2015). Although models of transitional care have been developed for other chronic health conditions, they rely on the patient to be an active participant in the process (Chenoweth et al. 2015; Farris et al. 2017). With the limited cognitive capacity of individuals with more severe dementia, this is not possible and often the responsibility to inform care transitions falls to the family (Chenoweth et al. 2015; Grealish et al. 2013). By customizing the EMR, the care plan and transition process can be adapted to enhance communication and better meet the specific needs and challenges of individuals with dementia.

There were several key factors that were important to successfully develop the dementia specific care plan and effectively inform the transition process. Other diagnosis specific care plans were subsequently developed within the organization using these principles. The key considerations for success in this work include: 
1. Establishment of a governance structure: This project had senior leadership support as a corporate goal and the commitment of key resources within the organization. The governance structure with Advisory Group Leadership, interprofessional working groups and subgroups expedited decision-making and the clear division of responsibilities. The inclusion of both administrative and clinical staff in the working groups and subgroups created a better understanding of the impact of EMR changes on clinicians.

2. The development of an interprofessional care plan placing the patient at the centre with the entire team working together to achieve the patient's goals: Having one interprofessional care plan also consolidates care plan information providing greater consistency in approaches to care by team members.

3. The integration of assessments and interventions into one care plan: By using an EMR, standardized tools were integrated into the assessment process and informed the identification of interventions. Creating frequencies (qshift, qweek, q28 days etc.) provided a structured process for re-assessment, tracking progress and re-evaluation of interventions. Prior to discharge, all the standardized assessments are repeated to provide another measure of patient progress and benchmarks that help to identify any changes that occur during the transition progress.

4. Strong communication between hospital and LTC facilities using both an EMR-based care plan and a transition process that includes conferences with patients, family/ SDM and community providers, and provides a comprehensive approach to the transfer of knowledge: In addition, transferring the interprofessional care plan alleviates the need to develop an entirely new care plan and promotes consistency in approach.

5. To align with Ontario Shores recovery philosophy, the care plan is written in the first person: By writing the care plan in the first person, people with dementia are given a voice in the identification of goals and interventions. It also allows staff to consider the daily care and management of BPSD from the patient's perspective. This reinforces the individualization of the care plan, an important part of the HQO Quality Standard.

There were some key challenges associated with the implementation of this project.

1. As with any new clinical process, change management is a vital component. Although staff was engaged early in the project and part of the change management process, competing projects often imposed increase work demands. In the case of this project, temporary increases in staffing levels were used to manage the change process.

2. Evaluating the effectiveness of transitional care is challenging because no objective measures are available (Coleman 2003). The team has now engaged our research department and will begin a study to measure the efficacy of incorporating video clips along with the care plan to support transitions in care.

\section{Conclusion}

This case study describes a mental health hospital's use of the EMR to develop a 
dementia care plan and transition process. As the healthcare system continues to find new ways to meet the needs of a growing population of adults with dementia, using technology is an important means to facilitate and inform care.

\section{References}

Chenoweth, L., A. Kable and D. Pond. 2015. "Research in Hospital Discharge Procedures Addresses Gaps in Care Continuity in the Community, but Leaves Gaping Holes for People with Dementia: A Review of the Literature." Australasian Journal on Ageing 34(1): 9-14. doi:10.1111/ajag.12205.

Coleman, E.A. 2003. "Falling Through the Cracks: Challenges and Opportunities for Improving Transitional Care for Persons with Continuous Complex Care Needs." Journal of the American Geriatrics Society 51(4): 549-55. doi:10.1046/j.1532-5415.2003.51185.x.

Dykes, P.C., L. Samal, M. Donahue, J.O. Greenberg, A.C. Hurley, O. Hasan et al. 2014. "A Patientcentered Longitudinal Care Plan: Vision Versus Reality." Journal of the American Medical Informatics Association 21(6): 1082-90. doi:10.1136/amiajnl-2013-002454.

Epstein-Lubow, G., A.T. Fulton, R. Gardner, S. Gravenstein and I.W. Miller. 2010.

"Post-hospital Transitions: Special Considerations for Individuals with Dementia." Medicine and Health, Rhode Island 93(4): 125.

Farris, G., M. Sircar, J. Bortinger, A. Moore, J.E. Krupp, J. Marshall et al. 2017. "Extension for Community Healthcare Outcomes-Care Transitions: Enhancing Geriatric Care Transitions Through a Multidisciplinary Videoconference." Journal of the American Geriatrics Society 65(3): 598-602. doi:10.1111/jgs.14690.

Fortinsky, R.H. and M. Downs. 2014. "Optimizing Person-Centered Transitions in the Dementia Journey: A Comparison of National Dementia Strategies." Health Affairs 33(4): 566-73. doi:10.1377/ hlthaff.2013.1304.

Grealish, L., M. Jamieson, J. Brown, B. Draper, B. Moore, M. Proctor and D. Gibson. 2013. The Interaction between Hospital and Communitybased Services for People with Dementia and their Carers. Sydney, AU: Alzheimer's Australia NSW.
Health Quality Ontario (HQO). 2016. Quality Standards Behavioural Symptoms of Dementia: Care for Patients in Hospitals and Residents in Long-Term Care Homes. Toronto, ON: Author. Kable, A., L. Chenoweth, D. Pond and C. Hullick. 2015. "Health Professional Perspectives on Systems Failures in Transitional Care for Patients With Dementia and their Carers: A Qualitative Descriptive Study.” BMC Health Services Research 15: 567. doi:10.1186/s12913015-1227-z.

National Collaborating Centre for Mental Health (UK). 2007. Dementia: a NICE-SCIE Guideline on Supporting People with Dementia and their Carers in Health and Social Care (Vol. 42, No. 42.). Leicester; London, UK: British Psychological Society.

Oades, L.G., T.P. Crowe and M. Nguyen. 2009. "Leadership Coaching Transforming Mental Health Systems from the Inside Out: The Collaborative Recovery Model as PersonCentred Strengths Based Coaching Psychology." International Coaching Psychology Review 4(1): 25-36.

Ray, C.A., V. Ingram and J. Cohen-Mansfield. 2015. "Systematic Review of Planned Care Transitions for Persons with Dementia." Neurodegenerative Disease Management 5(4): 317-31. doi:10.2217/ nmt.15.23.

Tsilimingras, D. and D.W. Bates. 2008. "Addressing Postdischarge Adverse Events: A Neglected Area." Joint Commission Journal on Quality and Patient Safety/Joint Commission Resources 34(2): 85.

Tsilimingras, D., B.-S. Kenneth and R. Brooks. 2009. "Postdischarge Adverse Events in the Elderly.” Journal of Patient Safety 5(4): 199-242. Van Poelgeest, R., J.-P. Heida, L. Pettit, R.J. de Leeuw and G. Schrijvers. 2015. "The Association between eHealth Capabilities and the Quality and Safety of Health Care in the Netherlands: Comparison of HIMSS Analytics EMRAM data with Elsevier's 'The Best Hospitals' Data.” Journal of Medical Systems 39(9): 90. 